\title{
Dynamique et physiopathologie des réseaux
}

\section{neuronaux}

(CIRB)

\section{Laurent Venance}

\section{(2) OpenEdition \\ Journals}

\section{Édition électronique}

URL : https://journals.openedition.org/annuaire-cdf/1083

DOI : 10.4000/annuaire-cdf. 1083

ISBN : 978-2-7226-0325-7

ISSN : 2109-9227

\section{Éditeur}

Collège de France

\section{Édition imprimée}

Date de publication : 1 avril 2013

Pagination : 884-886

ISBN : 978-2-7226-0198-7

ISSN : 0069-5580

\section{Référence électronique}

Laurent Venance, "Dynamique et physiopathologie des réseaux neuronaux », L'annuaire du Collège de France [En ligne], 112 | 2013, mis en ligne le 22 novembre 2013, consulté le 22 août 2022. URL : http:// journals.openedition.org/annuaire-cdf/1083; DOI : https://doi.org/10.4000/annuaire-cdf.1083 
Romain Gerbier (2008- 2011): Études structure-fonction du récepteur de l'apéline par modélisation moléculaire et mutagenèse dirigée. Recherche d'agonistes et/ou d'antagonistes de ce récepteur. Thèse de doctorat en sciences de l'université René Descartes Paris V, école doctorale «Médicament, toxicologie, chimie et environnement», co-direction avec Dr. X. Iturrioz, mention très honorable avec les félicitations du jury.

\title{
Dynamique et physiopathologie des réseaux neuronaux (CIRB)
}

\author{
Responsable : Laurent VENANCE \\ Équipe : C. Bosch, Y. CuI, B. Degos, E. Fino, Y. GioAnNi, M.-L. Kemel, S. Pérez, \\ S. Valtcheva, M. Vandecasteele, H. Xu, L. Venance
}

\section{RECHERCHE}

Nous étudions les modalités d'encodage de la mémoire au niveau des ganglions de la base, un ensemble de structures cérébrales sous-corticales impliquées dans le contrôle adaptatif du comportement et la mémoire procédurale. Le striatum, le principal noyau d'entrée des ganglions de la base, reçoit des afférences de l'ensemble des aires du cortex cérébral. Les neurones de sortie striataux, silencieux au repos, doivent, pour décharger, recevoir des signaux corticaux de fortes amplitudes et synchrones. Ainsi, les neurones striataux agissent comme des détecteurs de coïncidence de l'activité corticale : ils extraient du bruit de fond les informations corticales jugées pertinentes et ce, en fonction du contexte (motivation, attention, etc.). L'intégration des informations corticales est fortement modulée par les afférences dopaminergiques de la substance noire pars compacta. Le rôle prépondérant de la dopamine est dramatiquement illustré par la maladie de Parkinson causée par la dégénérescence des neurones dopaminergiques. L'apprentissage et la mémorisation sont principalement sous-tendus par des phénomènes de plasticité synaptique à long-terme qui soit augmentent l'efficacité synaptique (potentialisation à long-terme, LTP), soit la diminuent (dépression à long-terme, LTD). La connaissance des modalités de la plasticité cortico-striatale est fondamentale pour comprendre les mécanismes qui sous-tendent le rôle des ganglions de la base dans l'apprentissage procédural et leurs altérations en conditions physiopathologiques.

\section{La « spike-timing dependent plasticity » cortico-striatale}

Nous avons utilisé des protocoles de «spike-timing dependent plasticity » (STDP), récemment décrits comme étant plus physiologiques et considérés actuellement comme la loi «élémentaire» de plasticité synaptique. La STDP repose sur un paradigme d'apprentissage Hebbien et donc sur la quasi-coïncidence entre des stimulations pré- et post-synaptique. Ainsi, il a été décrit dans différentes structures 
qu'une stimulation pré-synaptique précédée de quelques millisecondes de la stimulation post-synaptique (répétées plusieurs dizaines de fois à $1 \mathrm{~Hz}$ ) induit une LTD, tandis qu'une stimulation post- puis pré-synaptique induit inversement une LTP. De manière surprenante, nous avons observé une orientation de la STDP « inverse » au niveau des neurones de sortie striataux: une stimulation post-pré induit une LTP et une stimulation pré-post induit une LTD. Cette plasticité « inverse » striatale était la première décrite chez les mammifères. Ceci indique une spécificité du codage au niveau de l'axe cortico-striatal. Afin de mieux caractériser cette STDP, nous avons étudié les voies de signalisation impliquées dans la mise en place de la STDP cortico-striatale. Contrairement au modèle «classique », nous avons montré que la LTP et la LTD sont sous-tendues par des détecteurs de coïncidence moléculaires différents. L'induction de la LTP est dépendante de l'activation des récepteurs NMDA post-synaptiques alors que la LTD dépend des endocannabinoïdes, plus précisément le 2-arachidonyl-glycerol qui agit sur les récepteurs cannabinoïdes de type 1 (CB1) pré-synaptiques. Nous avons mis en évidence un contrôle bimodal des endocannabinoides sur les plasticités à court et à long terme. De plus, nous avons montré que la sous-unité NR2B du récepteur NMDA contrôlait la fenêtre temporelle d'induction de la STDP. Cela illustre toute la complexité, et donc l'étendue des potentialités d'encodage, des plasticités cortico-striatales.

\section{Rôle des astrocytes dans le traitement des informations cortico-striatales}

Nous avons mis en évidence que les astrocytes, dans le cadre de la synapse tripartite, via la recapture du glutamate et du GABA, contrôlent la transmission et les plasticités à court- et long-terme cortico-striatales. Le concept de synapse tripartite (éléments neuronaux pré- et post-synaptiques et astrocytaires) a mis les cellules gliales au cœur des processus de traitement de l'information. Grâce à des enregistrements de double patch-clamp astrocyte-neurone, nous avons mis en évidence une activité électrogénique puissante au niveau des astrocytes striataux suite à une activation corticale, due aux transporteurs au glutamate et au GABA astrocytaire. Ainsi, un blocage de ces transporteurs aboutit à une baisse de l'efficacité de la transmission synaptique corticostriatale. Nous disposons d'un modèle d'étude absolument uniquement puisque les courants électroniques générés par les transports du glutamate et du GABA sont de très grandes amplitudes. Nous avons de plus observé des effets marqués des transports astrocytaires du glutamate et du GABA sur les phénomènes de plasticité à court- et long-terme (de type STDP) au niveau des neurones de sortie striataux. Ainsi, nous avons montré que les astrocytes par l'intermédiare du transport de neurotransmetteurs, participent activement à l'établissement des propriétés de détection de coïncidence opérée par les neurones striataux.

\section{La voie hyperdirecte de transmission de l'information cortex-ganglions de la base}

Si le striatum constitue la principale porte d'entrée des ganglions de la base, le noyau sous-thalamique (NST) constitue l'autre porte d'entrée. La voie cortex-NSTsubstance noire réticulée est appelé voie hyperdirecte, et son activation permet de mettre en pause les programmes en cours, afin de préparer la séquence suivante (via l'activation des voies directe et indirecte cortico-striatales). Dans la maladie de 
Parkinson, un traitement utilisé est la stimulation électrique profonde du NST. Nous avons démontré que les effets bénéfiques de la stimulation profonde proviendraient d'une balance excitation/inhibition engendrée non seulement par la stimulation électrique du NST mais aussi de fibres de passage issues du globus pallidus. Nous avons par ailleurs mis au point la première tranche de cerveau permettant de conserver intacte l'entièreté de la voie hyperdirecte et permettre ainsi des études électrophysiologiques, d'imagerie et pharmacologiques qui étaient impossible auparavant in vitro.

\section{Publications (2011-2012)}

Vandecasteele M. et Venance L., « Gap Junctions in the Basal Ganglia », in Dere E. (éd.), Gap Junctions in the Brain, Elsevier, 2012, sous presse.

Bosch C., Mailly P., Degos B., Deniau J.M. et Venance L., « Preservation of the hyperdirect pathway of basal ganglia in a rodent brain slice », Neuroscience, 215, 2012, 31-41.

Evans R.C., Morera-Herreras T., Cui Y., Du K., Sheehan T., Kotaleski J.H., Venance L. et Blackwell K.T., "The effects of NMDA subunit composition on calcium influx and spike timing-dependent plasticity in striatal medium spiny neurons », PLoS Comput. Biol., 8(4), 2012, e1002493.

Puente N., Cui Y., Lassalle O., Lafourcade M., Georges F., Venance L. ${ }^{2}$, Grandes P.* et Manzoni O.J.*, «Polymodal activation of the endocannabinoid system in the extended amygdala », Nature Neuroscience, 14(12), 2011, 1542-1547.

Vandecasteele M., Deniau J.M., Venance L., «Spike frequency adaptation is developmentally regulated in substantia nigra pars compacta dopaminergic neurons», Neuroscience, 29(192), 2011, 1-10.

Bosch C., Degos B., Deniau J.M. et Venance L., «Subthalamic nucleus high-frequency stimulation generates a concomitant synaptic excitation-inhibition in substantia nigra pars reticulata », J. Physiol., 589(Pt 17), 2011, 4189-4207.

Goubard V., Fino E. et Venance L., «Contribution of astrocytic glutamate and GABA uptake to corticostriatal information processing », J. Physiol., 589, 2011, 2301-2319.

Aliane V., Pérez S., Bohren Y., Deniau J.M. et Kemel M.L., «Key role of striatal cholinergic interneurons in processes leading to arrest of motor stereotypies », Brain, 134(Pt 1), 2011, 110-118.

Pandolfo P., Silveirinha V., dos Santos-Rodrigues A., Venance L., Ledent C., Takahashi R.N., Cunha R.A. et Köfalvi A., "Cannabinoids inhibit the synaptic uptake of adenosine and dopamine in the rat and mouse striatum », Eur. J. Pharmacol., 655(1-3), 2011, $38-45$.

Fino E. et Venance L., «Spike-timing dependent plasticity in striatal interneurons », Neuropharmacology, 60(5), 2011, 780-788.

2. Co-auteurs séniors. 